\title{
INTRALABORATORY COMPARISON IN THE NOTCH MEASUREMENT OF CHARPY TEST SPECIMENS
}

\author{
FABRICIO, Daniel Antonio Kapper ${ }^{1 *}$; ROCHA, Cláudia Lisiane Fanezi da²; REGULY, Afonso ${ }^{3}$; \\ STROHAECKER, Telmo Roberto ${ }^{4}$
}

1,2,3,4 Universidade Federal do Rio Grande do Sul, Escola de Engenharia, Laboratório de Metalurgia Física.

Av. Osvaldo Aranha, 99/610, CEP 90035-190, Porto Alegre - RS, Brasil

(fone: +55 513308 4251; fax: +55 513308 3988)

\author{
${ }^{*}$ Autor correspondente \\ e-mail: daniel.fabricio@ufrgs.br
}

Received 27 March 2015; received in revised form 2 July 2015; accepted 14 August 2015

\section{RESUMO}

O entalhe de corpos de prova Charpy possui tolerâncias restritas indicadas na norma ASTM E23, as quais devem ser analisadas antes do ensaio de impacto. Visando avaliar a confiabilidade da medição, um estudo intralaboratorial foi conduzido. Os resultados indicaram que o processo possui boa reprodutibilidade, sendo o sistema validado nessas condições.

Palavras-chave: Ensaio de impacto Charpy, Comparação intralaboratorial, Validação do entalhe.

\section{ABSTRACT}

The notch of Charpy test specimens have tight tolerances according to ASTM E23 standard. These should be considered before conducting impact tests. To evaluate the measurement reliability, an intralaboratory study was conducted. The results indicate a good reproducibility of the measurement process, validating the system under these conditions.

Keywords: Charpy impact testing, Intralaboratory comparison, Notch validation.

\section{INTRODUÇÃO}

$\begin{array}{rcc}\text { O ensaio de impacto é um ensaio } \\ \text { dinâmico } & \text { utilizado para análise do }\end{array}$ comportamento de fratura de materiais. Esse ensaio fornece a energia necessária para fraturar uma amostra padronizada de acordo com as normas ASTM E23 e BS EN ISO 148-1. Entretanto, deve ser enfatizado que a energia de impacto absorvida pelo corpo de prova fornece uma informação qualitativa de tenacidade à fratura, sendo que seus resultados podem ser somente comparados entre si ou com um valor determinado em uma especificação (GARCIA et al., 2012).

Os corpos de prova de ensaio Charpy possuem um entalhe que simula um defeito em serviço. O entalhe a ser usinado possui tolerâncias restritas segundo indicado na norma de ensaio ASTM E23 (ASTM, 2012), as quais são relacionadas ao raio, ângulo e profundidade do mesmo. Caso estas características não sejam atendidas, o corpo de prova é considerado inadequado pela norma, pois grandes variações serão observadas no resultado final do ensaio.

A norma ABNT NBR ISO/IEC 17025 estabelece requisitos gerais para a competência técnica de laboratórios de ensaios e calibrações. Entre os requisitos, está a garantia da qualidade das análises realizadas: a norma exige que o laboratório tenha e execute procedimentos para monitorar a qualidade das medições realizadas, 
devendo ser aplicadas técnicas estatísticas para a análise dos resultados, com base em critérios de aceitação pré-definidos (ABNT, 2005).

A análise de variância (ANOVA) é uma metodologia estatística que permite avaliar a significância de diversos fatores e interações. A ANOVA de um fator compara as variâncias entre os valores médios de uma variável a diferentes níveis de um fator, resultando em um valor de razão entre variâncias denominado $F_{\text {calculado. }}$ Em seguida, compara-se o valor $F_{\text {calculado }}$ com um valor tabelado da distribuição de Fisher-Snedecor $\left(\mathrm{F}_{\mathrm{ta}-}\right.$ belado), utilizando-se um nível de significância específico, usualmente 5\% (RIBEIRO e CATEN, 2012). Se o valor $F_{\text {calculado }}$ for maior que o valor $F_{\text {ta- }}$ belado, então a variabilidade provocada pelo termo correspondente é significativa frente ao erro residual.

O teste do Erro Normalizado $\left(E_{n}\right)$ testa a compatibilidade dos resultados das medições do laboratório com respeito a um valor de referência. $O$ valor de $E_{n}$ pode ser calculado pela Equação 1 (INMETRO, 2010). O resultado é considerado aceitável quando o erro normalizado é menor ou igual à unidade.

$E_{n}=\frac{\left|X_{1}-X_{2}\right|}{\sqrt{u_{1}^{2}+u_{2}^{2}}}$

A Equação 1 é uma relação entre a diferença de médias do resultado de dois parâmetros analisados $\left(\mathrm{X}_{1}-\mathrm{X}_{2}\right)$ e a raiz quadrada da soma das incertezas expandidas dos resultados obtidos para o primeiro parâmetro $\left(u_{1}\right)$ e para o segundo parâmetro $\left(u_{2}\right)$.

Tendo em vista a importância do monitoramento da qualidade das medições previsto na ABNT NBR ISO/IEC 17025, esse trabalho visa realizar uma análise intralaboratorial com o objetivo de avaliar a confiabilidade do processo de medição do entalhe em corpos de prova tipo Charpy.

\section{MATERIAL E MÉTODOS}

Foram recebidos 8 corpos de prova para ensaio de Charpy obtidos a partir de dois diferentes materiais em aço (Material I e Material II), sendo 4 corpos de prova de cada um. Os corpos de prova eram do tipo tamanho reduzido, com dimensão de valor nominal de $55 \times 5 \times 5$ $\mathrm{mm}$ (Figura 1) e entalhe do tipo A (Charpy-V).
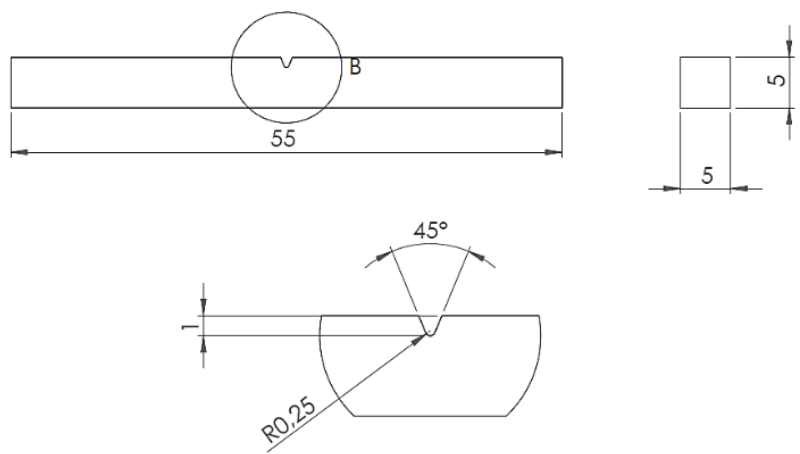

Figura 1. Corpo de prova Charpy de dimensão reduzida com entalhe tipo $A$

O entalhe usinado deve seguir as tolerâncias dimensionais descritas na norma ASTM E23 (ASTM, 2007), segundo indicado na Tabela 1.

Tabela 1. Tolerâncias dimensionais do entalhe em corpos de prova Charpy com entalhe tipo $A$

\begin{tabular}{c|c|c|c}
\hline $\begin{array}{c}\text { Tolerânci } \\
\text { a }\end{array}$ & $\begin{array}{c}\text { Raio } \\
{[\mathbf{m m}]}\end{array}$ & $\begin{array}{c}\text { Profundidade } \\
{[\mathbf{m m}]}\end{array}$ & $\begin{array}{c}\text { Ângulo } \\
{\left[{ }^{\circ}\right]}\end{array}$ \\
\hline Mínimo & 0,225 & 0,975 & 44 \\
\hline Nominal & 0,250 & 1,000 & 45 \\
\hline Máximo & 0,275 & 1,025 & 46 \\
\hline
\end{tabular}

Com o auxílio de um projetor de perfil (Figura 2), foram analisados raio, profundidade $e$ ângulo do entalhe de cada um dos 8 corpos de prova. Essa medição foi realizada por dois diferentes operadores (Operador A e Operador B), seguindo o mesmo procedimento. O equipamento utilizado possui resolução de $0,5 \mu \mathrm{m}$ e capacidade de medição de $150 \mathrm{~mm}$ no eixo horizontal e $50 \mathrm{~mm}$ no eixo vertical. Para medição de ângulo, a resolução é de $00^{\circ} 00^{\prime} 01^{\prime \prime}$, com capacidade de até $360^{\circ}$. 


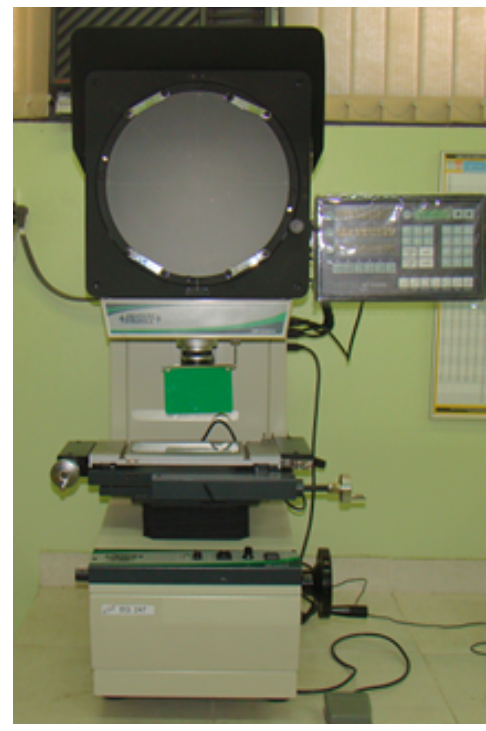

Figura 2. Projetor de perfil

De posse das medições dimensionais realizadas, procedeu-se à análise dos dados através dos métodos estatísticos do erro normalizado e ANOVA, considerando o operador como fator de estudo. O critério de aceitação para o método do erro normalizado foi $E_{n} \leq 1$, e para o método ANOVA foi $F_{\text {calculado }}<F_{\text {critico, }}$, conforme indicado na literatura (RIBEIRO e CATEN, 2012; COSTA et al., 2008; MARQUES et al., 2008; COUTO et al., 2009; BRIONIZIO et al., 2005).

O cálculo do erro normalizado depende da incerteza de medição expandida, conforme indicado na Equação 1. Nesse trabalho, o cálculo da incerteza associada à média de cada variável foi realizado utilizando o Guia para Expressão da Incerteza de Medição (JCGM, 2008), considerando como fontes de incerteza a resolução do equipamento, o desvio padrão de repetitividade e a incerteza herdada do certificado de calibração do projetor de perfil.

Os corpos de prova foram retirados de dois Materiais diferentes (Material I e Material II); portanto, as análises através da ANOVA e do erro normalizado foram realizadas para os dois grupos de corpos de prova separadamente.

\section{RESULTADOS E DISCUSSÃO}

As medidas do entalhe dos corpos de prova, realizadas no projetor de perfil pelos Operadores A e B, são apresentadas na Tabela 2.
A partir dos valores das médias e das incertezas de medição expandidas, procedeu-se ao cálculo do erro normalizado entre os operadores, obtendo-se os valores $E_{n}$ apresentados na Tabela 3.

Tabela 3. Erro normalizado para o dimensional dos corpos de prova

\begin{tabular}{c|c|c|c}
\hline Material & $\begin{array}{c}\text { Profundidad } \\
\mathbf{e}\end{array}$ & Ângulo & Raio \\
\hline I & 0,07 & 0,66 & 0,09 \\
\hline II & 0,17 & 0,60 & 0,06 \\
\hline
\end{tabular}

Complementarmente, foi realizada ANO$V A$ para o fator operador a um nível de significância de 5\%. A Tabela 4 exemplifica uma das análises de variância realizadas (medição da profundidade do entalhe dos corpos de prova do Material I).

A Tabela 5 compila os valores de $F_{\text {calculado }}$ e $F_{\text {tabelado }}$ obtidos a partir das análises de variância realizadas.

A partir dos dados da Tabela 3, nota-se que os valores de erro normalizado foram inferiores a 1,00 para a medida do raio, profundidade $e$ ângulo do entalhe. Além disso, pela Tabela 5, nota-se que todos os valores de $F_{\text {calculado }}$ foram inferiores aos valores de $F_{\text {tabelado }}$ a um nível de significância de $5 \%$. Assim, pelos dois métodos, os resultados foram considerados aceitáveis, sendo a dispersão entre os operadores inferior ao resíduo aleatório.

Foi observado que alguns valores medidos (Tabela 2) não estão de acordo com as tolerâncias estabelecidas na norma ASTM E23 (Tabela 1). Isso, no entanto, não influencia no objetivo geral do trabalho de estudar a reprodutibilidade do método de medição.

Após este estudo, foi implementado um gabarito (template) com o objetivo de facilitar a validação do entalhe de acordo com as tolerâncias especificadas na norma ASTM E23.

\section{CONCLUSÕES}

Visando avaliar a confiabilidade do processo de medição do entalhe em corpos de prova tipo Charpy, medidas dimensionais foram realizadas em um projetor de perfil por dois operadores e uma análise intralaboratorial foi conduzida através dos métodos do erro 
normalizado e análise de variância.

Os resultados indicaram que a dispersão entre as medições realizadas pelos diferentes operadores foi inferior ao resíduo relacionado à repetitividade das medidas, tanto pelo método do erro normalizado quanto por ANOVA. Assim, conclui-se que o processo de medição do entalhe através de projetor de perfil possui boa reprodutibilidade, sendo esse sistema de medição validado para essa aplicação.

\section{AGRADECIMENTOS}

Os autores agradecem ao MSc. Eng. Vagner Machado Costa, ao João Vítor Herrmann e à Agnes Ágata Fróes Martins pelo apoio na execução desse trabalho.

\section{REFERÊNCIAS}

1. ABNT. NBR ISO/IEC 17025: Requisitos gerais para a competência de laboratórios de ensaio e calibração. Associação Brasileira de Normas Técnicas: Rio de Janeiro, 2005.

2. ASTM E23-12c: Standard Test Methods for Notched Bar Impact Testing of Metallic Materials. ASTM International: West Conshohocken, 2012.

3. Brionizio J.; Lima, S.; Mainier, F. Comparação intralaboratorial em Higrometria. Encontro para a Qualidade de Laboratórios - ENQUALAB, São Paulo, 2005.

4. Costa, P.; Muniz, B.; Machado, R.; Koch, C.; Barros, W. Comparação entre métodos para medição de impressões de dureza Brinell e Vickers. $1^{\circ}$ Congresso Internacional de Metrologia Mecânica CIMMEC, Rio de Janeiro, 2008.

5. Couto, P.; Oliveira, L.; Oliveira, J.; Silva, W.; $\quad$ Soares, R. Comparação intralaboratorial do Lapre-Inmetro na faixa de medição de $1,4 \mathrm{kPa}$ a $7 \mathrm{MPa}$. V Congresso Brasileiro de Metrologia, Salvador, 2009.

6. Garcia, A.; Spim, J. A.; Santos, C. A. Ensaio dos Materiais. 2 ed., LTC: Rio de Janeiro, 2012.

7. INMETRO. DOQ-CGCRE-008: Orientação sobre Validação de Métodos Analíticos. Coordenação Geral de Acreditação, Revisão 03, Fev. 2010.

8. JCGM. Evaluation of measurement data Guide to the expression of uncertainty in measurement (GUM), 1st ed. Joint Committee for Guides in Metrology: 2008.

9. Marques, A.; Alves, J.; Costa, P.; Barros, W. Comparação interlaboratorial de métodos de verificação de máquinas de medir por coordenadas (MMC). $1^{\circ}$ Congresso Internacional de Metrologia Mecânica - CIMMEC, Rio de Janeiro, 2008.

10. Ribeiro, J. L. D.; Caten, C. S. T. Cartas de Controle para Variáveis, Cartas de Controle para Atributos, Função de Perda Quadrática, Análise de Sistemas de Medição. FEENG/UFRGS: Porto Alegre, 2012. 
Tabela 2. Medidas dimensionais dos corpos de prova

\begin{tabular}{ccccccc}
\hline \multicolumn{7}{c}{ Material I } \\
\hline Corpo de & Profundidade [mm] & \multicolumn{1}{c}{ Ângulo [ ${ }^{\circ}$ ] } & \multicolumn{2}{c}{ Raio [mm] } \\
\cline { 2 - 7 } Prova & Op. A & Op. B & Op. A & Op. B & Op. A & Op. B \\
\hline I-1 & 0,894 & 0,936 & 45,390 & 47,301 & 0,267 & 0,273 \\
I-2 & 0,886 & 0,885 & 44,430 & 45,211 & 0,280 & 0,293 \\
I-3 & 1,060 & 1,082 & 45,440 & 46,125 & 0,285 & 0,277 \\
I-4 & 1,073 & 1,075 & 45,500 & 48,103 & 0,281 & 0,278 \\
Média & 0,98 & 0,99 & 45,19 & 46,69 & 0,28 & 0,28 \\
Desvio Padrão & 0,10 & 0,10 & 0,51 & 1,27 & 0,01 & 0,01 \\
Incerteza & 0,17 & 0,16 & 0,84 & 2,11 & 0,01 & 0,01 \\
\hline \multicolumn{7}{c}{ Material II } \\
Corpo de & Profundidade [mm] & Ângulo [ ${ }^{\circ}$ ] & Raio [mm] \\
Prova & Op. A & Op. B & Op. A & Op. B & Op. A & Op. B \\
\hline II-1 & 0,935 & 1,112 & 44,060 & 45,335 & 0,267 & 0,232 \\
II-2 & 1,110 & 0,920 & 46,470 & 47,080 & 0,259 & 0,283 \\
II-3 & 1,037 & 0,954 & 46,230 & 49,523 & 0,261 & 0,263 \\
II-4 & 1,072 & 1,042 & 45,340 & 48,560 & 0,267 & 0,268 \\
Média & 1,04 & 1,01 & 45,52 & 47,62 & 0,26 & 0,26 \\
Desvio Padrão & 0,08 & 0,09 & 1,09 & 1,82 & 0,00 & 0,02 \\
Incerteza & 0,12 & 0,14 & 1,80 & 3,02 & 0,01 & 0,04 \\
\hline
\end{tabular}

Tabela 4. ANOVA do fator operador para a profundidade do entalhe dos corpos de prova do Material

\begin{tabular}{c|c|c|c|c|c}
\hline $\begin{array}{c}\text { Fonte da } \\
\text { Variação }\end{array}$ & $\begin{array}{c}\text { Soma dos } \\
\text { Quadrado } \\
\mathbf{s}\end{array}$ & $\begin{array}{c}\text { Graus de } \\
\text { Liberdad } \\
\mathbf{e}\end{array}$ & $\begin{array}{c}\text { Média dos } \\
\text { Quadrado } \\
\mathbf{s}\end{array}$ & $\mathbf{F}_{\text {calculado }}$ & $\mathbf{F}_{\text {tabelado }}$ \\
\hline Operador & 0,001 & 1 & 0,001 & 0,051 & 5,987 \\
\hline Resíduo & 0,061 & 6 & 0,010 & & \\
\hline Total & 0,061 & 7 & & & \\
\hline
\end{tabular}

Tabela 5. Resultados da ANOVA para o dimensional dos corpos de prova

\begin{tabular}{c|c|c|c|c|c|c}
\hline \multirow{2}{*}{ Material } & \multicolumn{2}{|c|}{ Profundidade } & \multicolumn{2}{c|}{$\hat{\text { Ângulo }}$} & \multicolumn{2}{c}{ Raio } \\
\cline { 2 - 7 } & $\mathbf{F}_{\text {calculado }}$ & $\mathbf{F}_{\text {tabelado }}$ & $\mathbf{F}_{\text {calculado }}$ & $\mathbf{F}_{\text {tabelado }}$ & $\mathbf{F}_{\text {calculado }}$ & $\mathbf{F}_{\text {tabelado }}$ \\
\hline $\mathrm{I}$ & 0,051 & 5,987 & 4,746 & 5,987 & 0,105 & 5,987 \\
\hline II & 0,300 & 5,987 & 3,893 & 5,987 & 0,037 & 5,987 \\
\hline
\end{tabular}

PERIÓDICO TCHÊ QUÍMICA • www.periodico.tchequimica.com • Vol. 12 N. 24

- ISSN 1806-0374 (impresso) • ISSN 1806-9827 (CD-ROM) • ISSN 2179-0302 (meio eletrônico)

(C) 2015. Porto Alegre, RS. Brasil

The Periódico Tchê Química (ISSN: 1806-0374; 2179-0302) is an open-access journal since 2004. Journal DOI: 10.52571/PTQ. http://www.tchequimica.com. This text was introduced in this file in 2021 for compliance reasons.

(๑) The Author(s)
OPEN ACCESS. This article is licensed under a Creative Commons Attribution 4.0 (CC BY 4.0) International License, which permits use, sharing, adaptation, distribution, and reproduction in any medium or format, as long as you give appropriate credit to the original author(s) and the source, provide a link to the Creative Commons license, and indicate if changes were made. The images or other third-party material in this article are included in the or exceeds the permitted use, you will need to obtain permission directly from the copyright holder. To view a copy of this license, visit htp://creativecommons org/licenses/by/4.0/: 